\title{
The utility of independent component analysis and machine learning in the identification of the amyotrophic lateral sclerosis diseased brain
}

\author{
Robert C. Welsh ${ }^{1,2}{ }^{*}$, Laura M. Jelsone-Swain ${ }^{1}$ and Bradley R. Foerster ${ }^{1,3}$ \\ 1 Department of Radiology, University of Michigan, Ann Arbor, MI, USA \\ ${ }^{2}$ Department of Psychiatry, University of Michigan, Ann Arbor, MI, USA \\ ${ }^{3}$ Ann Arbor VA Healthcare System, Ann Arbor, MI, USA
}

\section{Edited by:}

Veronika Schöpf, Medical University

Vienna, Austria

Reviewed by:

Federica Agosta, Vita-Salute San Raffaele University, Italy

Christian Rummel, University Institute for Diagnostic and Interventional

Neuroradiology, Switzerland

${ }^{*}$ Correspondence:

Robert C. Welsh, Department of Radiology, University of Michigan,

Medical Science I, Room 3208C, 1301

Catherine Street, Ann Arbor, MI

48109, USA

e-mail: rcwelsh@med.umich.edu
Amyotrophic lateral sclerosis (ALS) is a devastating disease with a lifetime risk of $\sim 1$ in 2000. Presently, diagnosis of ALS relies on clinical assessments for upper motor neuron and lower motor neuron deficits in multiple body segments together with a history of progression of symptoms. In addition, it is common to evaluate lower motor neuron pathology in ALS by electromyography. However, upper motor neuron pathology is solely assessed on clinical grounds, thus hindering diagnosis. In the past decade magnetic resonance methods have been shown to be sensitive to the ALS disease process, namely: resting-state connectivity measured with functional MRI, cortical thickness measured by high-resolution imaging, diffusion tensor imaging (DTI) metrics such as fractional anisotropy and radial diffusivity, and more recently magnetic resonance spectroscopy (MRS) measures of gamma-aminobutyric acid concentration. In this present work we utilize independent component analysis to derive brain networks based on resting-state functional magnetic resonance imaging and use those derived networks to build a disease state classifier using machine learning (support-vector machine). We show that it is possible to achieve over $71 \%$ accuracy for disease state classification. These results are promising for the development of a clinically relevant disease state classifier. Future inclusion of other MR modalities such as high-resolution structural imaging, DTI and MRS should improve this overall accuracy.

Keywords: independent component analysis, support vector machine, resting-state functional connectivity, amyotrophic lateral sclerosis, machine learning, disease-state classification

\section{INTRODUCTION}

Amyotrophic lateral sclerosis (ALS) is a progressive neurodegenerative disease involving the motor cortex, corpus callosum, cortical spinal tract, and spinal anterior horn neurons, and presents with upper motor neuron and lower motor neuron signs (Ghadge et al., 2003; Turner et al., 2009). The disease can have a highly variable presentation and can be challenging to diagnose, which can have significant implications for the patients as the median survival time is between 2 and 4 years (Beghi et al., 2006). There is no definitive diagnostic test for ALS. The diagnosis relies on the clinical examination to detect upper and lower motor neuron signs in multiple body segments (Brooks et al., 2000) along with symptom progression. Unfortunately, there is on average a 1-year delay between onset of symptoms and diagnosis for this rapidly progressive disease (Zoccolella et al., 2006), which precludes timely intervention with emerging disease-modifying treatments. The development of reliable diagnostic and prognostic biomarkers would represent a significant advance in the clinical work-up of ALS (Karitzky and Ludolph, 2001; Cudkowicz et al., 2004; Turner et al., 2009).

Conventional magnetic resonance imaging provides limited and potentially inconsistent information describing ALS patients (Cheung et al., 1995; Hofmann et al., 1998; Comi et al., 1999;
Chan et al., 2003). Therefore, there has been great interest in using advanced neuroimaging modalities to establish markers of ALS. Although techniques such as voxel-based morphometry (Roccatagliata et al., 2009), resting-state functional connectivity (Mohammadi et al., 2009; Jelsone-Swain et al., 2010; Verstraete et al., 2011; Agosta et al., 2013), magnetic resonance spectroscopy (Foerster et al., 2012a), and diffusion tensor imaging (Filippini et al., 2010) have demonstrated differences between groups of ALS patients and healthy controls (HC), few studies have investigated diagnostic test accuracy measures (Turner and Modo, 2010; Foerster et al., 2012b).

Functional connectivity is a relatively new and powerful advanced neuroimaging method to evaluate regional brain interactions (establishing neural networks) that occur when a subject is not performing an explicit task (Biswal et al., 1997; Lowe et al., 1998; Jelsone-Swain et al., 2010). Alterations of brain networks have been seen in diseases such as Alzheimer's disease (Greicius et al., 2004), schizophrenia (Welsh et al., 2010), depression (Zeng et al., 2012), obsessive compulsive disorder (Stern et al., 2011), as well as ALS (Mohammadi et al., 2009; Jelsone-Swain et al., 2010; Verstraete et al., 2010, 2011; Douaud et al., 2011; Agosta et al., 2013). In particular, there is evidence of extensive brain network alterations due to the ALS disease process, such as those affecting 
the default-mode network (Mohammadi et al., 2009), motor networks (Douaud et al., 2011), and fronto-parietal networks (Agosta et al., 2013).

Statistical image analysis that can incorporate the entirety of a brain image can have an advantage over massively parallel univariate techniques (Wang and Summers, 2012). Machine-learning methods integrate a potentially large number of observables (that is, variables or features, and in the example of functional connectivity the feature space spans the number of connection strengths/edges derived from each resting-state time-series) into a coherent analysis that leverages the combined space of the features into an increase of detection power (Chen et al., 2008). Machine-learning methods using functional connectivity data have been applied to classify disease state such as in Alzheimer's disease (Magnin et al., 2008; Orrù et al., 2012), depression (Craddock et al., 2009), and other psychiatric diseases (Orrù et al., 2012), and therefore could also be applied to ALS. To meet this important unmet need, we have explored the utility of machinelearning methodology to analyze resting-state functional magnetic resonance imaging (fMRI) data for ALS disease classification.

\section{MATERIALS AND METHODS PARTICIPANTS}

We recruited 32 patients diagnosed with ALS and 31 age and gender matched healthy controls (HCs). The ALS patients were recruited through the University of Michigan Motor Neuron Disease Clinic in the Department of Neurology at the University of Michigan. HC participants were recruited through local advertising and web portals. This study was approved by the University of Michigan Institutional Review Board. The participants gave informed consent prior to the MRI examination. All participants in this cohort underwent MRI examination, which included resting-state fMRI. All ALS participants had date of symptom onset recorded as well as disease severity at time of scan assessed by the ALS Functional Rating Scale, revised version (ALSFRS-R) (Cedarbaum et al., 1999). The maximum score of the ALSFRS-R is 48 , with lower scores indicating increased physical disability.

\section{MAGNETIC RESONANCE ACOUISITION Image acquisition}

All scanning took place on a GE 3T Excite 2 magnet (General Electric, Milwaukee, WI, USA). All participants had high-resolution anatomic $\mathrm{T}_{1}$-weighted imaging (spoiled-gradient-recall, SPGR). High-resolution images were collected with a $256^{2}$ matrix, $220 \mathrm{~mm}$ FOV, and $1.0 \mathrm{~mm}$ slice thickness) and resting-state fMRI. $\mathrm{T}_{2}{ }^{*}$ time-series data were acquired parallel to the AC-PC axis using a reverse-spiral $k$-space readout. A total of $240 \mathrm{~T}_{2}{ }^{*}$-weighted volumes were collected during each scanning session (repetition time, $\mathrm{TR}=2 \mathrm{~s} ; 40$-slice volumes; $3 \mathrm{~mm}$ slice thickness, no skip; echo time, $\mathrm{TE}=30 \mathrm{~ms} ; 64 \times 64$ matrix; field-of-view FOV $=220 \mathrm{~mm}$ ).

\section{Functional connectivity}

Resting-state time-series data were pre-processed similarly to Welsh et al. (2010). We used an in-house pre-preprocessing method which uses both FSL 4.1.9 (Jenkinson et al., 2012) and SPM8 (release 4667). Time-series data were preprocessed in the following steps: slice-time corrected (FSL), motion corrected
(FSL), and normalized to MNI space (SPM8/VBM8). Time-series data were resampled to $3 \mathrm{~mm}$ voxel resolution and isotropically smoothed with a 5-mm Gaussian kernel. A mask of white-matter was derived from the SPGR during the spatial normalization step using VBM8. To minimize partial volume effects, the resulting mask was eroded three times over with FSL. A similarly derived cerebral spinal fluid (CSF) mask was also created, however, due to variance in ventricular size across subjects the CSF mask was only eroded once. Prior to independent components analysis (ICA) data were further filtered: (1) global signal normalization was performed (Chang and Glover, 2009; Fox et al., 2009); (2) motion parameters (translation and rotation) were regressed from the time-series data; (3) voxel time-courses were then extracted from white-matter and CSF masks and analyzed with principle components analysis (PCA), following Behzadi et al. (2007) the top five PCA components were then used to regress out systematic variance due to physiological noise; (4) data were then band-pass filtered (fast-Fourier transform) in the $0.01-0.10-\mathrm{Hz}$ range (Cordes et al., 2001).

The resulting time-series data for each subject was then independently analyzed with ICA using FSL/Melodic (Beckmann and Smith, 2004). The number of components was not specified as the number was best determined by Melodic (Beckmann and Smith, 2004) using the Minimum Description Length algorithm (Rissanen, 1978). The ICA analysis produced between 15 and 40 ICA spatial components and corresponding temporal modes ${ }^{1}$.

Next, we used the spatial templates from the networks defined in Smith et al. (2009). We took the top 10 templates defined from their BrainMap analysis ${ }^{2}$ in the 20-component ICA scheme, thresholding each map at (component magnitude) $>3.0$. These template network maps were then used to identify the corresponding resting-state network (RSN) in our analysis. Assignment of a particular network to a component was done by maximizing the overall match for all 10 RSNs following the procedure of Greicius et al. (2004). A score was calculated for each network for the best matching ICA spatial component by taking the average of the in-map spatial component weight minus the average out-of-map spatial component weight. We required that a component could only be used once and if there was one component best matched to two or more RSNs, then all possible combinations were searched to get an overall best RSNs match for that subject.

In order to provide properly scaled data to the support vector machine, a correlation map for any particular RSN was created by calculating the correlation coefficient for each voxel in the RSN with the associate ICA time-course, after all other ICA time-courses had been regressed from the voxel timeseries.

For this work we explored the utility of disease state classification based upon the networks that have been shown to be altered in ALS: DMN, Motor, and Fronto-Parietal. Additionally, given the observed $\sim 35 \%$ cognitive impairment in ALS (JelsoneSwain et al., 2012) we also included the frontal executive network. In the Smith et al. (2009) nomenclature these are RSNs: RSN04,

\footnotetext{
${ }^{1}$ Mean number of components for ALS was $27+/-7$ and the mean number of components for $\mathrm{HC}$ was $24+/-4$.

${ }^{2}$ http://www.fmrib.ox.ac.uk/analysis/brainmap+rsns/
} 
RSN06, RSN07 ${ }^{3}$, RSN08, RSN09, RSN10. The selected networks are shown in Figure 1.

\section{SUPPORT VECTOR MACHINE}

Current implementations of support vector machines were first formulated by Cortes and Vapnik (1995). Briefly, a support vector machine is a supervised learning formalism that allows for complex solutions of discrimination classification. Typically an array of measures is carried out for each instance of a class. In our study measures of connectivity (the array of measures) are determined for each participant (each participant being an instance/observation) in the study. Unlike a massively parallel univariate analysis carried out between two groups, support vector machine formalism examines all measures simultaneously to determine a hyperplane in the space defined by the array of

${ }^{3}$ We visually inspected each RSN template prior to selection. By using the "atlas" tool in FSL we determined that RSN07 also included portions of the motor system (pre-central gyrus), therefore we included it as a relevant network. measures that may separate the two groups. Using the notation of Guyon and Elisseeff (2003):

$$
D(\mathbf{x})=\mathbf{w} \mathbf{x}+b
$$

using a training data set, the decision boundary is optimized to give the weights $\boldsymbol{w}$. At the testing phase the decision solution for a given observation $\mathbf{x}$ of the array of measures can be calculated with the weight vector $\boldsymbol{w}$ determined by the SVM, and $b$ is a bias value also determined by the SVM. When $D(\mathbf{x})<0$, then $\mathbf{x}$ belongs to the first class, while $D(\mathbf{x})>0$ indicates membership in the second class. Inherently, the decision is a binary one. Further formalism of the SVM methodology can be found in Cortes and Vapnik (1995).

As a simple example we illustrate this concept with Figure 2. In both examples each observation is characterized by two metrics. The boundary of the first is easily derived, but the boundary of the second that maximally discriminates between the two groups can take a highly complex form, even in this two-dimensional example.

We utilized the support vector machine (libsvm version 3.17) implementation of Chang and Lin (2011) and we opted to use

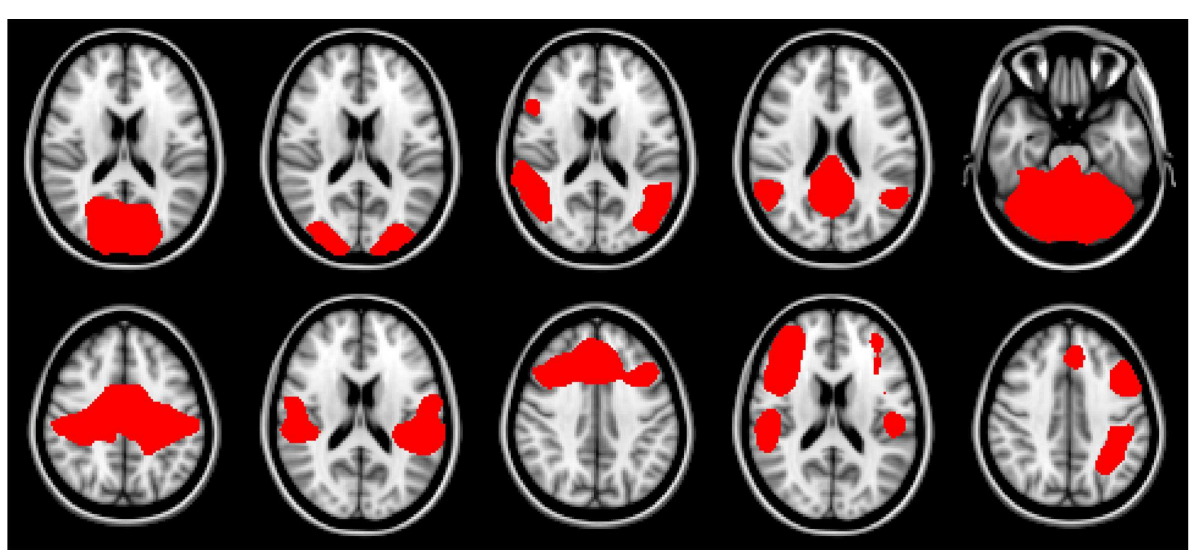

FIGURE 1 | Resting-state network templates corresponding to networks 1 through 10 from Smith et al. (2009). RSNs are numbered 1 through 10, with upper left being 1 , and lower right be number 10 . As in Smith et al. (2009) networks are as follows: 1-3: visual, 4: default mode, 5: cerebellum, 6: sensorimotor, 7: auditory, 8: executive, 9-10 fronto-parietal.

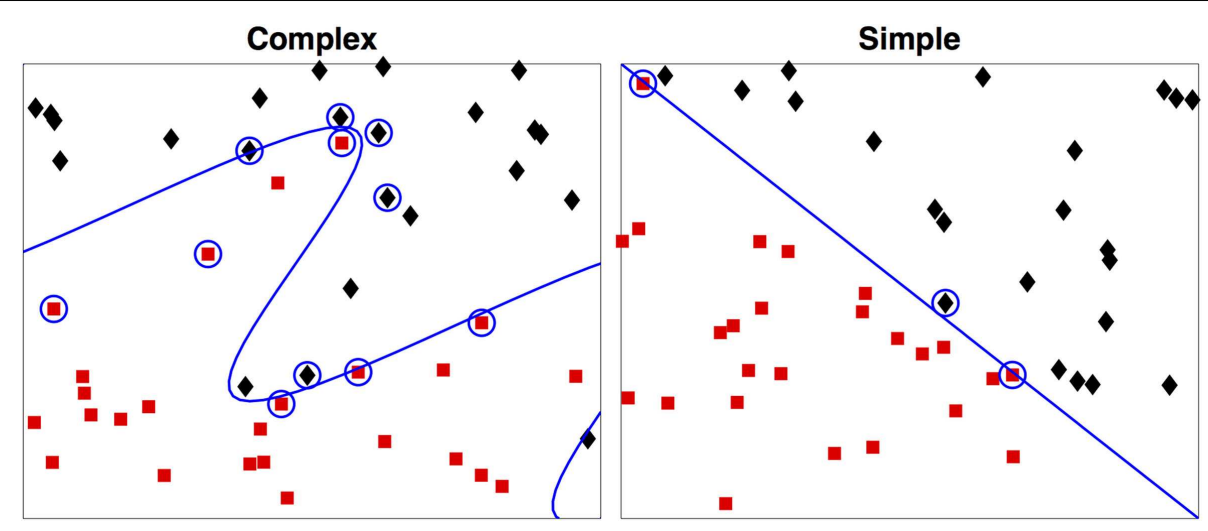

FIGURE 2 |Two-dimensional examples of a simple and a complex support vector machine solution. The two-class membership is indicated by color. The support vectors are indicated by the circles with the boundary defined by $D(\mathbf{x})=0$. During testing only the support vectors are used in determination of the class for the test case. 
the linear kernel. Given the observations in the literature for compromises related to ALS in a variety of resting-state networks, we built feature-vectors of correlation coefficients from the following RSNs: default mode (Mohammadi et al., 2009), motor areas (Verstraete et al., 2010, 2011; Douaud et al., 2011), and left and right frontal-parietal regions (Agosta et al., 2013).

We used leave-one-out-cross-validation (LOOCV) (Burges, 1998) for calculation of SVM accuracy. The overall scheme is shown in Figure 3. We also examined the efficacy of simple feature filtering by including those features from a network that passed a two-tailed liberal statistically significant group difference of $p \leq 0.05$ (uncorrected) (Craddock et al., 2009). We performed a bootstrap (Jiang and Simon, 2007) on the LOOCV (100 bootstraps) and calculated the class prediction for each test case in the LOOCV as a continuous variable by averaging the 100 binary decision results. This average class prediction then allowed for the calculation of a receiver-operator curve (ROC). To estimate the variance on the area-under-the-curve (AUC) of the ROC we performed a bootstrap ROC.

Final classification accuracy was defined as the:

$$
\frac{N_{\text {Correct }}^{\mathrm{ALS}}+N_{\text {Correct }}^{\mathrm{HC}}}{N_{\text {Total }}^{\mathrm{ALS}}+N_{\text {Total }}^{\mathrm{HC}}}
$$

with $N_{\text {Total }}^{\text {ALS }}=32$ and $N_{\text {Total }}^{\mathrm{HC}}=31 . N_{\text {Correct }}^{\text {ALS }}$ and $N_{\text {Correct }}^{\text {ALS }}$ being the number of correctly SVM classified ALS and HC participants. To assess performance of the SVM against a typical univariate method, we followed methods by Fair et al. (2007) and calculated the number of nodes (Sporns, 2009) (voxels) present for a subject in the given network surpassing a $Z$-score of $0.10,0.15,0.20$, 0.25 , and 0.30 . The number of nodes by subject was then used to calculate a univariate ROC.

\section{RESULTS DEMOGRAPHICS}

A total of 32 individuals with ALS were enrolled in our study. Our main objective for HCs was to match for age. Mean ALS age was $58.4 \pm 6.6$ years and our HCs were aged $56.9 \pm 5.0$ and there was no significant difference in age (two-sample $t$-test $p=0.319)$. We did have a slight imbalance in gender matching, with ALS male/female $=21 / 11$, and HC male/female $=16 / 15$. However there was no age by gender bias, $p>0.05$. Mean time since onset of symptoms for ALS was $1.8 \pm 1.4$ years with a range of $0.4-6.0$ years. ALSFRS-R average score was $38.2 \pm 5.7$ with a range of 25-46. The ALSFRS-R score and time of scan since symptom onset distributions are show in Figure 4.

\section{ICA GROUP VALIDATION}

To demonstrate that the template matching succeeded, we calculated typical resting-state group analyses: subject correlation maps for each RSN were converted to $Z$-scores and entered into random effects analyses by group. Statistical images for the default mode (RSN04) and the primary motor network (RSN06) are shown in Figure 5.

\section{SUPPORT VECTOR MACHINE RESULTS}

In this survey of classification performance the SVM achieved $71.5 \%$ accuracy for determination of disease state as either ALS or healthy. This maximal classification accuracy came from a combined use of the default-mode network (RSN04) and the primary motor network (RSN06). For this combination the fraction of correctly classified ALS and HC was $N_{\text {Correct }}^{\text {ALS }}=23$ and $N_{\text {Correct }}^{\mathrm{HC}}=22$. The SVM classification and univariate classification ROCs are shown in Figure 6. The bootstrap calculated AUC and variance was AUC $=0.716 \pm 0.047$. The univariate AUC was $\mathrm{AUC}=0.544 \pm 0.008$. To test for a classification bias due to

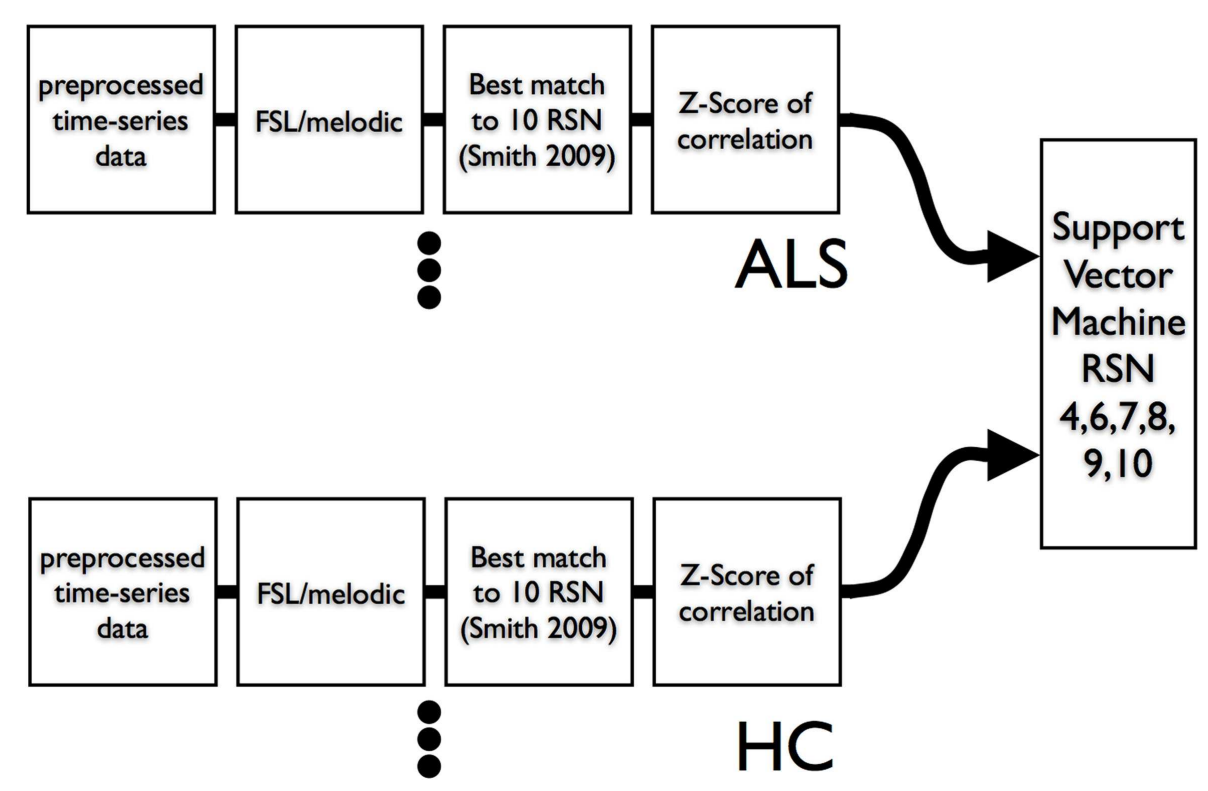

FIGURE 3 | Data flow of resting-state times series into ICA and then into SVM. A leave-one-out-cross-validation was utilized to assess SVM classification accuracy. 

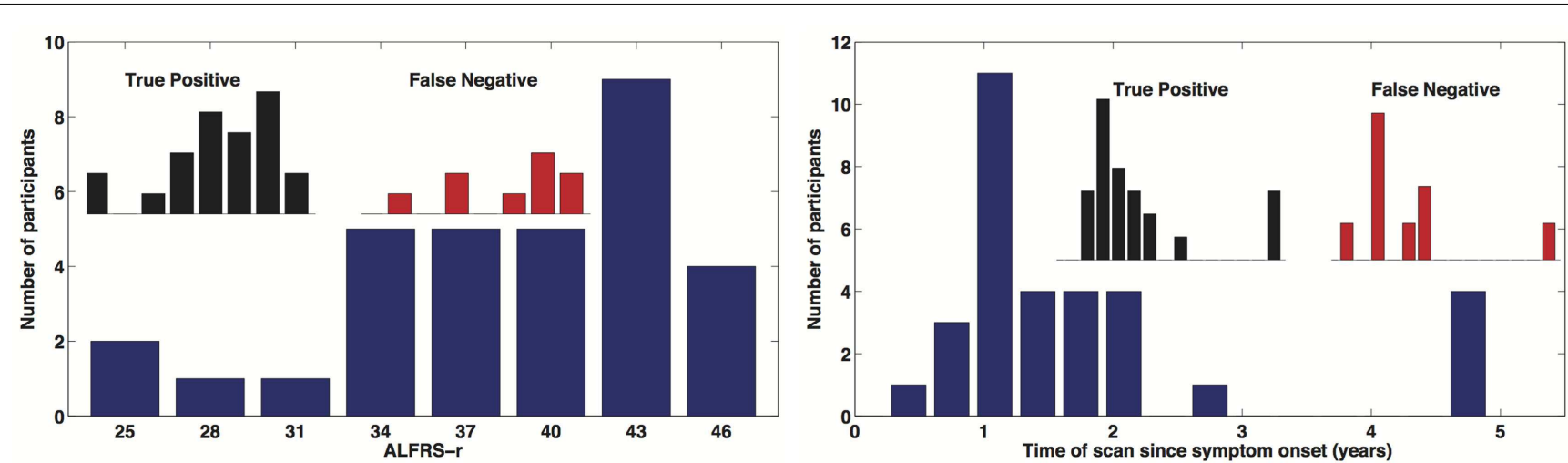

FIGURE 4 | Distribution of time of scan since symptom onset and observed distribution of ALSFRS-r in our ALS participant cohort. Inserted distributions are for the true-positive and false-negative classified groups.

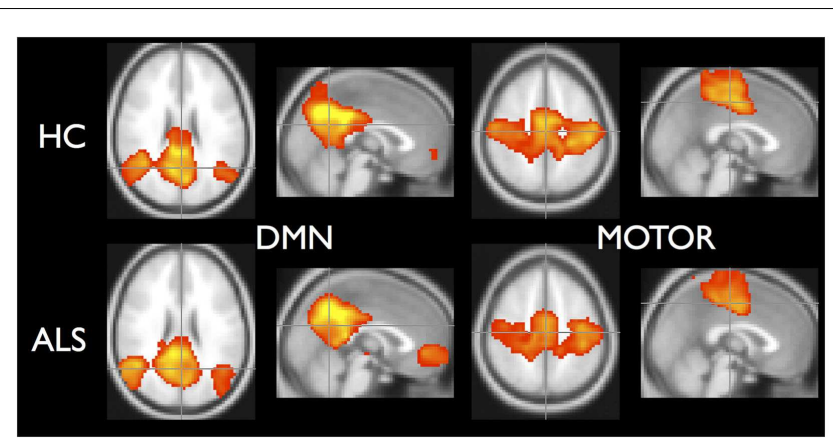

FIGURE 5 | Random effects analysis for default-mode network and motor network. Healthy controls are in top row and ALS are in bottom row. Statistical maps thresholded for $t \geq 3.5$ for illustrative purposes.

ALSFRS-R or time-of-scan-since-symptom-onset (ONSET) we did a post hoc examination of the ALSFRS-R score and ONSET for those ALS participants that were accurately classified as ALS and those incorrectly classified as healthy. We also tested disease progression rate [defined as (48-ALSFRS-R)/ONSET] between these groups. We performed a non-parametric KolmogorovSmirnov (Chakravarti et al., 1967) test to assess if these values were drawn from the same or different parent distribution. Comparison of ALSFRS-R, ONSET, and progression rate between true-positive and false-negative groups revealed no significant differences $(p=0.306, p=0.744$, and $p=0.372$ respectively). The ALSFRS-R and ONSET distributions for true positives and false negatives are shown in Figure 4.

\section{DISCUSSION}

Our work combined resting-state connectivity [derived from ICA (Beckmann and Smith, 2004)] and machine learning (Chang and Lin, 2011) to explore their utility for ALS disease-state prediction. ICA reliably identified well established (Damoiseaux et al., 2006; Smith et al., 2009) RSNs in our ALS and HC cohorts. By using a subset of these networks that have been shown to be altered by the ALS disease process (Mohammadi et al., 2009; Jelsone-Swain et al., 2010; Verstraete et al., 2010; Douaud et al., 2011; Agosta et al.,

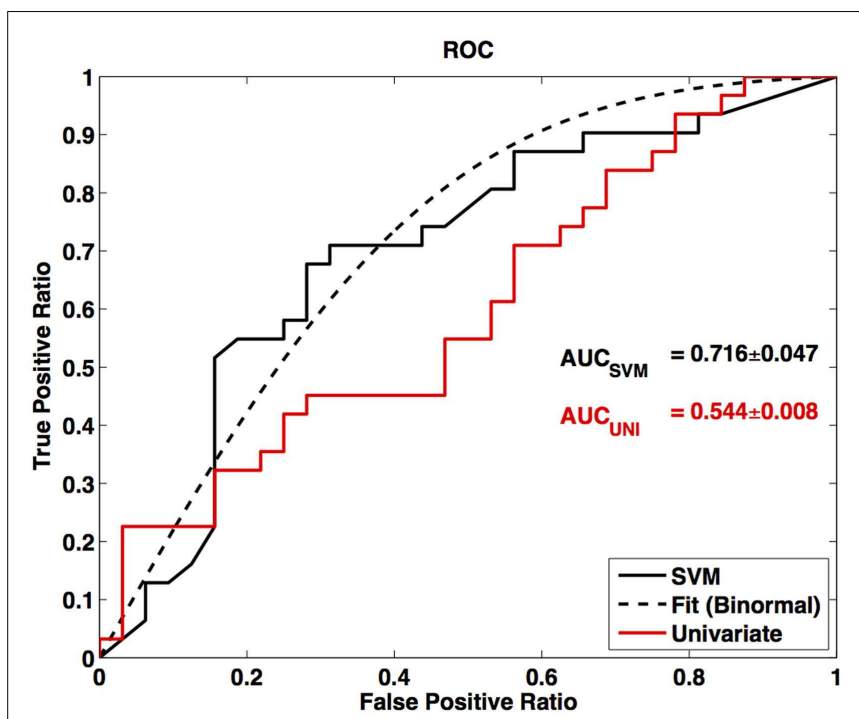

FIGURE 6 | Receiver-operator curve for LOOCV SVM and ROC for univariate node counting. Smooth curve is a binormal (Cai and Moskowitz, 2004) fit to the LOOCV SVM ROC. AUC is calculated from binormal fit.

2013), in conjunction with machine learning (as implemented with a support vector machine), we have shown that machine learning has modest disease classification accuracy using restingstate $\mathrm{fMRI}$ data. The AUC of the SVM indicates better performance than the univariate classifier. In schizophrenia SVM derived classifications have been found to be between $\sim 62$ and $~ 85 \%$ (Tang et al., 2012; Yu et al., 2013) using whole brain connectivity. Using a specific and more finite number of network nodes Craddock et al. (2009) achieved 62\% classification accuracy in depression with comparable $t$-test filtering.

Although extra motor regions have been implicated in ALS using other advanced neuroimaging methods such as diffusion tensor imaging (DTI), it is important to note that the motor networks had significant contribution to the SVM state classification. Though not presently recognized as a resting-state network showing alteration, the executive control network also contributes to 
the disease state classification. The classification sensitivity to the executive network could be due to the $\sim 35 \%$ observed cognitive impairment seen in ALS (Rippon et al., 2006; Jelsone-Swain et al., 2012). Our findings demonstrate the power of multivariate techniques such as machine learning. We have shown there can exist significant systematic differences when the network is considered as a whole even though there can be a lack of statistically significant differences in specific node or edge-wise comparisons, such as in counting of significant nodes in a network (Sporns, 2009).

Seeley et al. (2009) suggested that disease state can be classified by functional network metrics derived from resting-state measurements using fMRI. Until recently the vast majority of the resting-state fMRI literature tested for statistically significant group differences with a voxel-wise approach (Smith, 2012). Much of that work was done with either seed based analysis or ICA (Fox et al., 2005; Damoiseaux et al., 2006) to determine these brain networks. The voxel-wise approach requires a spatial coherence in change due to the disease process, but also requires an observable change that meets statistical significance after correction for multiple comparisons (Marchini and Presanis, 2004). Although in our previous approach of investigating group differences, we relaxed this condition of spatially coherent change in the network by examining distribution differences of network metrics (correlation coefficient) (Jelsone-Swain et al., 2010). More recent group comparison approaches have taken on graph theoretical methodology (Cabral et al., 2011) to address questions of group differences.

In a multivariate approach, the data are used coherently to assess significance between groups. This approach is readily extended to build a decision algorithm to determine group membership based on the full suite of variables under consideration. The decision algorithm can be trained with an independent dataset and then assessed for accuracy through the use of an independent testing dataset. Indeed this is the operational approach of machine learning (Vatolkin et al., 2012). Essentially a mathematical decision boundary can be derived in the space of the suite of variables. This boundary is derived to maximize the separation of the groups to be classified [though due to noise in real systems one would not expect 100\% separation (Wang and Summers, 2012)].

Discovering differences in brain metrics between two cohorts leads to a better understanding of the effect that a disease process has on a brain (Bandettini, 2012), such as an aberration in the motor network in ALS (Jelsone-Swain et al., 2010; Verstraete et al., 2010, 2011; Douaud et al., 2011). However, with increased understanding of observed changes, patterns can be revealed. These group differences can manifest patterns that can then be identified through machine-learning algorithms, more specifically in regard to identifying group membership. Eventually this can lead to the use of brain metrics to classify between two brain activity states (Laconte et al., 2005; De Martino et al., 2008; LaConte, 2011), or between states of diseased and healthy (Orrù et al., 2012). Thus, invoking multivariate techniques can lead to better state differentiation than differentiation based on voxel-wise or edge-wise univariate comparisons.

Advanced neuroimaging techniques, specifically resting-state fMRI, DTI and voxel-based morphometry, generate a large number of potentially useful data points. It is becoming increasingly clear that more conventional univariate brain analysis techniques used in concert with single modality imaging techniques do not provide sufficient disease discrimination in ALS. For example, a meta-analysis of DTI data results indicates only modest diagnostic test accuracy in ALS (Foerster et al., 2012b). As a result there is increased interest in applying more advanced brain mapping statistical techniques in ALS, including the implementation of machine-learning methods to analyze advanced MRI data in an effort to develop an imaging "fingerprint" of disease. The results presented here point to the potential utility of machinelearning methods to classify disease status in ALS using imaging data sets with a large number of variables. Additional research efforts are required to further explore this approach including combining different advanced neuroimaging approaches using machine-learning methods. In addition to resting-state fMRI the modalities to be included should be: DTI (Chapman et al., 2013), high-resolution structural imaging (Grosskreutz et al., 2006), and magnetic resonance spectroscopy (Foerster et al., 2012a) as put forth by Turner et al. (2009). Furthermore, given the heterogenous presentation of ALS, which can lead to clinical diagnostic uncertainly, it would be warranted to apply SVM classification methods and advanced neuroimaging techniques to a cohort of individuals at first presentation with neurological symptoms. The imaging should occur prior to a definitive diagnosis of ALS and follow the individuals longitudinally. Given the differential nature of disease diagnosis, future studies should also include ALS mimics, that is, other neurodegenerative diseases with overlapping symptom presentation, to fully explore the power of classification schemes (Turner and Modo, 2010). The true diagnostic utility of such a classifier would be in providing input into the clinical process with the resulting goal of shortening the duration between symptom presentation and final diagnosis of ALS.

\section{LIMITATIONS}

There are of course limitations to our study. First, ALS is a highly divergent disease process with highly varying progression paths. Certainly, utilizing a larger cohort of individuals with ALS and a larger cohort of HCs would lead to a better definition of classifiers. Though the ALS disease process has a quite divergent nature we have built our classifier decision from two classes. Another approach would be to build a single state (one-class) classifier (Manevitz and Yousef, 2002). Under those conditions, the question would be, "Does the test case belong to the one-class classifier?" Our approach has also only included a single category of metrics, namely resting-state derived brain networks. Other MRI modalities have also been shown to be sensitive to the ALS disease process, such as cortex thinning (Roccatagliata et al., 2009; Turner et al., 2009), increased radial diffusivity (RD) and decreased fractional anisotropy (FA) (Wang and Melhem, 2005; Schimrigk et al., 2007; Filippini et al., 2010; Foerster et al., 2012b), and more recently decreased gamma-aminobutyric acid (GABA) concentration in the motor cortex (Foerster et al., 2012a). We do note that this is the first application of machine learning for the classification of disease status in ALS using MRI data ${ }^{4}$. Construction of a complex

${ }^{4}$ A pubmed.org search of the title/abstract terms [("ALS" or "amyotrophic lateral sclerosis") and ("support vector machine" or "SVM")] yields no imaging literature. 
differential diagnosis classification scheme first has to demonstrate distinction between well-defined classes such as definite ALS and HCs. As such, this current work is exploratory in nature but demonstrates the clear promise of such techniques to continue in the near future.

\section{CONCLUSION}

Resting-state functional connectivity reveals intrinsic networks in the human brain. These networks can be viewed as patterns that are a manifestation of the state of the brain including altered network patterns present in disease (Seeley et al., 2009). By applying multivariate pattern classification methodology we have demonstrated that machine-learning methodology (support vector machine) in conjunction with brain networks derived from resting-state fMRI can be used to classify a diseased brain (ALS) from a healthy brain.

\section{REFERENCES}

Agosta, F., Canu, E., Valsasina, P., Riva, N., Prelle, A., Comi, G., et al. (2013). Divergent brain network connectivity in amyotrophic lateral sclerosis. Neurobiol. Aging 34, 419-427. doi:10.1016/j.neurobiolaging.2012. 04.015

Bandettini, P. A. (2012). Twenty years of functional MRI: the science and the stories. Neuroimage 62, 575-588. doi:10.1016/j.neuroimage.2012.04. 026

Beckmann, C. F., and Smith, S. M. (2004). Probabilistic independent component analysis for functional magnetic resonance imaging. IEEE Trans. Med. Imaging 23, 137-152. doi:10.1109/TMI.2003.822821

Beghi, E., Logroscino, G., Chiò, A., Hardiman, O., Mitchell, D., Swingler, R., et al. (2006). The epidemiology of ALS and the role of populationbased registries. Biochim. Biophys. Acta 1762, 1150-1157. doi:10. 1016/j.bbadis.2006.09.008

Behzadi, Y., Restom, K., Liau, J., and Liu, T. T. (2007). A component based noise correction method (CompCor) for BOLD and perfusion based fMRI. Neuroimage 37, 90-101. doi:10.1016/j.neuroimage.2007.04. 042

Biswal, B. B., Van Kylen, J., and Hyde, J. S. (1997). Simultaneous assessment of flow and BOLD signals in resting-state functional connectivity maps. NMR Biomed. 10, 165-170. doi:10.1002/(SICI)10991492(199706/08)10:4/5<165::AIDNBM454>3.0.CO;2-7

Brooks, B. R., Miller, R. G., Swash, M., and Munsat, T. L. (2000). El Escorial revisited: revised criteria for the diagnosis of amyotrophic lateral sclerosis. Amy otroph. Lateral Scler. Other Motor Neuron Disord. 1, 293-299. doi:10. 1080/146608200300079536
Burges, C. (1998). A tutorial on support vector machines for pattern recognition. Data Min. Knowl. Discov. 2, 121-167. doi:10.1023/A:1009715923555

Cabral, J., Hugues, E., Sporns, O., and Deco, G. (2011). Role of local network oscillations in resting-state functional connectivity. Neuroimage 57, 130-139. doi:10.1016/j.neuroimage.2011.04. 010

Cai, T., and Moskowitz, C. S. (2004). Semi-parametric estimation of the binormal ROC curve for a continuous diagnostic test. Biostatistics 5, 573-586. doi:10.1093/biostatistics/kxh009

Cedarbaum, J. M., Stambler, N., Malta, E., Fuller, C., Hilt, D., Thurmond, B., et al. (1999). The ALSFRSR: a revised ALS functional rating scale that incorporates assessments of respiratory function. BDNF ALS Study Group (Phase III). J. Neurol. Sci. 169, 13-21. doi:10.1016/S0022510X(99)00210-5

Chakravarti, I. M., Laha, R. G., and Roy, J. (1967). Handbook of Methods of Applied Statistics, Vol. 1. New York: John Wiley and Sons. 392-394.

Chan, S., Kaufmann, P., Shungu, D. C., and Mitsumoto, H. (2003). Amyotrophic lateral sclerosis and primary lateral sclerosis: evidence-based diagnostic evaluation of the upper motor neuron. Neuroimaging Clin. N. Am. 13, 307-326. doi:10.1016/ S1052-5149(03)00018-2

Chang, C., and Glover, G. H. (2009). Effects of model-based physiological noise correction on default mode network anti-correlations and correlations. Neuroimage 47, 1448-1459. doi:10.1016/j.neuroimage.2009.05. 012

Chang, C.-C., and Lin, C.-J. (2011). LIBSVM. ACM Trans.

Additional research efforts are required to validate our findings as well as to investigate the added diagnostic utility of including other MR modalities in the setting of ALS using machine-learning methods.

\section{ACKNOWLEDGMENTS}

We would like to thank Dr. Kirsten Gruis and the ALS Clinic/Motor Neuron Clinic at the University of Michigan for their invaluable assistance in recruitment of ALS patients. We would like to thank Rebecca Hovatter and Nick Rademacher for study coordination. We thank MR research technologist Keith Newnham for assisting in collection of the MR data. This work has been supported by Basic Radiological Sciences Seed Grant (Robert C. Welsh) and NIH/NINDS R01-NS052514 (Robert C. Welsh).

Intell. Syst. Technol. 2, 1-27. doi:10.1145/1961189.1961199

Chapman, M. C., Jelsone-Swain, L., Johnson, T. D., Gruis, K. L., and Welsh, R. C. (2013). Diffusion tensor MRI of the corpus callosum in amyotrophic lateral sclerosis. J. Magn Reson. Med. (in press).

Chen, S.-H., Sun, J., Dimitrov, L., Turner, A. R., Adams, T. S., Meyers, D. A., et al. (2008). A support vector machine approach for detecting gene-gene interaction. Genet. Epidemiol. 32, 152-167. doi:10.1002/gepi.20272

Cheung, G., Gawel, M. J., Cooper, P. W., Farb, R. I., Ang, L. C., and Gawal, M. J. (1995). Amyotrophic lateral sclerosis: correlation of clinical and MR imaging findings. Radiology 194, 263-270.

Comi, G., Rovaris, M., and Leocani, L. (1999). Review neuroimaging in amyotrophic lateral sclerosis. Eur. J. Neurol. 6, 629-637. doi:10.1046/j.14681331.1999.660629.x

Cordes, D., Haughton, V. M., Arfanakis, K., Carew, J. D., Turski, P. A., Moritz, C. H., et al. (2001). Frequencies contributing to functional connectivity in the cerebral cortex in "restingstate" data. AJNR Am. J. Neuroradiol. 22, 1326-1333.

Cortes, C., and Vapnik, V. (1995). Support vector machine. Mach. Learn. 20, 273-297. doi:10.1023/A:1022627411411

Craddock, R. C., Holtzheimer, P. E. III, Hu, X. P., and Mayberg, H. S. (2009) Disease state prediction from resting state functional connectivity. Magn. Reson. Med. 62, 1619-1628. doi:10.1002/mrm.22159

Cudkowicz, M., Qureshi, M., and Shefner, J. (2004). Measures and markers in amyotrophic lateral sclerosis. NeuroRx 1, 273-283. doi:10.1602/neurorx.1.2.273
Damoiseaux, J. S., Rombouts, S. A. R. B., Barkhof, F., Scheltens, P., Stam, C. J., Smith, S. M., et al. (2006). Consistent resting-state networks across healthy subjects. Proc. Natl. Acad. Sci. U.S.A. 103, 13848-13853. doi:10.1073/ pnas.0601417103

De Martino, F., Valente, G., Staeren, N., Ashburner, J., Goebel, R., and Formisano, E. (2008). Combining multivariate voxel selection and support vector machines for mapping and classification of fMRI spatial patterns. Neuroimage 43, 44-58. doi:10.1016/j.neuroimage.2008.06. 037

Douaud, G., Filippini, N., Knight, S., Talbot, K., and Turner, M. R. (2011). Integration of structural and functional magnetic resonance imaging in amyotrophic lateral sclerosis. Brain 134, 3470-3479. doi:10.1093/brain/ awr279

Fair, D. A., Dosenbach, N. U. F., Church, J. A., Cohen, A. L., Brahmbhatt, S. Miezin, F. M., et al. (2007). Development of distinct control networks through segregation and integration. Proc. Natl. Acad. Sci. U.S.A. 104, 13507-13512. doi:10.1073/ pnas.0705843104

Filippini, N., Douaud, G., Mackay, C. E., Knight, S., Talbot, K., and Turner, M. R. (2010). Corpus callosum involvement is a consistent feature of amyotrophic lateral sclerosis. Neurology 75, 1645-1652. doi:10.1212/ WNL.0b013e3181fb84d1

Foerster, B. R., Callaghan, B. C., Petrou, M., Edden, R. A. E., Chenevert, T. L., and Feldman, E. L. (2012a). Decreased motor cortex-aminobutyric acid in amyotrophic lateral sclerosis. Neurology 78, 1596-1600. doi:10. 1212/WNL.0b013e3182563b57 
Foerster, B. R., Dwamena, B. A., Petrou, M., Carlos, R. C., Callaghan, B. C., and Pomper, M. G. (2012b). Diagnostic accuracy using diffusion tensor imaging in the diagnosis of ALS: a meta-analysis. Acad. Radiol. 19, 1075-1086. doi:10.1016/j.acra.2012.04.012

Fox, M. D., Snyder, A. Z., Vincent, J. L., Corbetta, M., Van Essen, D. C., and Raichle, M. E. (2005). The human brain is intrinsically organized into dynamic, anticorrelated functional networks. Proc. Natl. Acad. Sci. U.S.A. 102, 9673-9678. doi:10.1073/pnas.0504136102

Fox, M. D., Zhang, D., Snyder, A. Z., and Raichle, M. E. (2009). The global signal and observed anticorrelated resting state brain networks. J. Neurophysiol. 101, 3270-3283. doi:10.1152/ jn. 90777.2008

Ghadge, G. D., Slusher, B. S., Bodner, A., Canto, M. D., Wozniak, K., Thomas, A. G., et al. (2003). Glutamate carboxypeptidase II inhibition protects motor neurons from death in familial amyotrophic lateral sclerosis models. Proc. Natl. Acad. Sci. U.S.A. 100, 9554-9559. doi:10.1073/pnas. 1530168100

Greicius, M. D., Srivastava, G., Reiss, A. L., and Menon, V. (2004). Default-mode network activity distinguishes Alzheimer's disease from healthy aging: evidence from functional MRI. Proc. Natl. Acad. Sci. U.S.A. 101, 4637-4642. doi:10.1073/pnas.0308627101

Grosskreutz, J., Kaufmann, J., Frädrich, J., Dengler, R., Heinze, H.-J., and Peschel, T. (2006). Widespread sensorimotor and frontal cortical atrophy in amyotrophic lateral sclerosis. BMC Neurol. 6:17. doi:10.1186/1471-2377-6-17

Guyon, I., and Elisseeff, A. (2003). An introduction to variable and feature selection. J. Mach. Learn. Res. 3, 1157-1182.

Hofmann, E., Ochs, G., Pelzl, A., and Warmuth-Metz, M. (1998). The corticospinal tract in amyotrophic lateral sclerosis: an MRI study. Neuroradiology 40, 71-75. doi:10.1007/s002340050543

Jelsone-Swain, L., Persad, C., Votruba, K. L., Weisenbach, S. L., Johnson, T. D., Gruis, K. L., et al. (2012). The relationship between depressive symptoms, disease state, and cognition in amyotrophic lateral sclerosis. Front. Psychol. 3:542. doi:10.3389/ fpsyg.2012.00542

Jelsone-Swain, L. M., Fling, B. W., Seidler, R. D., Hovatter, R., Gruis, K., and Welsh, R. C. (2010). Reduced interhemispheric functional connectivity in the motor cortex during rest in limb-onset amyotrophic lateral sclerosis. Front. Syst. Neurosci. 4:158. doi:10.3389/fnsys.2010.00158

Jenkinson, M., Beckmann, C. F., Behrens, T. E. J., Woolrich, M. W., and Smith, S. M. (2012). FSL. Neuroimage $62,782-790$. doi:10.1016/j

Jiang, W., and Simon, R. (2007). A comparison of bootstrap methods and an adjusted bootstrap approach for estimating the prediction error in microarray classification. Stat. Med. 26, 5320-5334. doi:10.1002/sim.2968

Karitzky, J., and Ludolph, A. C. (2001). Imaging and neurochemical markers for diagnosis and disease progression in ALS. J. Neurol. Sci. 191, 35-41. doi:10.1016/S0022$510 \mathrm{X}(01) 00628-1$

Laconte, S., Strother, S., Cherkassky, V., Anderson, J., and Hu, X. (2005). Support vector machines for temporal classification of block design fMRI data. Neuroimage 26, 317-329. doi:10.1016/j.neuroimage.2005.01. 048

LaConte, S. M. (2011). Decoding fMRI brain states in real-time. Neuroimage 56, 440-454. doi:10. 1016/j.neuroimage.2010.06.052

Lowe, M. J., Mock, B. J., and Sorenson, J. A. (1998). Functional connectivity in single and multislice echoplanar imaging using resting-state fluctuations. $\mathrm{Neu}$ roimage $7,119-132$. doi:10.1006/ nimg. 1997.0315

Magnin, B., Mesrob, L., Kinkingnéhun S., Pélégrini-Issac, M., Colliot, O., Sarazin, M., et al. (2008). Support vector machine-based classification of Alzheimer's disease from wholebrain anatomical MRI. Neuroradiology 51, 73-83. doi:10.1007/s00234008-0463-x

Manevitz, L. M., and Yousef, M. (2002). One-class SVMs for document classification. J. Mach. Learn. Res. 2, 139-154.

Marchini, J., and Presanis, A. (2004). Comparing methods of analyzing fMRI statistical parametric maps. Neuroimage 22, 1203-1213. doi:10. 1016/j.neuroimage.2004.03.030

Mohammadi, B., Kollewe, K., Samii, A., Krampfl, K., Dengler, R., and Münte, T. F. (2009). Changes of resting state brain networks in amyotrophic lateral sclerosis. Exp. Neurol. 217, 147-153.

Orrù, G., Pettersson-Yeo, W., Marquand, A. F., Sartori, G., and Mechelli, A. (2012). Using support vector machine to identify imaging biomarkers of neurological and psychiatric disease: a critical review. $\mathrm{Neu}$ rosci. Biobehav. Rev. 36, 1140-1152. doi:10.1016/j.neubiorev.2012.01.004

Rippon, G. A., Scarmeas, N., Gordon, P. H., Murphy, P. L., Albert, S. M., Mitsumoto, H., et al. (2006). An observational study of cognitive impairment in amyotrophic lateral sclerosis. Arch. Neurol. 63, 345-352. doi:10.1001/archneur.63.3.345

Rissanen, J. (1978). Modeling by shortest data description. Automatica 14, 465-471. doi:10.1016/00051098(78)90005-5

Roccatagliata, L., Bonzano, L., Mancardi, G., Canepa, C., and Caponnetto, C. (2009). Detection of motor cortex thinning and corticospinal tract involvement by quantitative MRI in amyotrophic lateral sclerosis. Amyotroph. Lateral Scler. 10, 47-52. doi:10.1080/17482960802267530

Schimrigk, S. K., Bellenberg, B., Schlüter, M., Stieltjes, B., Drescher R., Rexilius, J., et al. (2007). Diffusion tensor imaging-based fractional anisotropy quantification in the corticospinal tract of patients with amyotrophic lateral sclerosis using a probabilistic mixture model. AJNR Am. J. Neuroradiol. 28, 724-730.

Seeley, W. W., Crawford, R. K., Zhou, J., Miller, B. L., and Greicius, M. D. (2009). Neurodegenerative diseases target large-scale human brain networks. Neuron 62, 42-52. doi:10.1016/j.neuron.2009.03.024

Smith, S. M. (2012). The future of FMRI connectivity. Neuroimage 62, 1257-1266. doi:10.1016/ j.neuroimage.2012.01.022

Smith, S. M., Fox, P. T., Miller, K. L., Glahn, D. C., Fox, P. M. Mackay, C. E., et al. (2009). Correspondence of the brain's functional architecture during activation and rest. Proc. Natl. Acad. Sci. U.S.A. 106, 13040-13045. doi:10.1073/pnas.0905267106

Sporns, O. (2009). "The human connectome: linking structure and function in the human brain," in Diffusion MRI, eds H. Johansen-Bers and T. Behrens (San Diego: Elsevier), 309-332.

Stern, E. R., Welsh, R. C., Fitzgerald, K. D., Gehring, W. J., Lister, J. J., Himle, J. A., et al. (2011). Hyperactive error responses and altered connectivity in ventromedial and frontoinsular cortices in obsessive-compulsive disorder. Biol. Psychiatry 69, 583-591. doi:10.1016/j.biopsych.2010.09.048

Tang, Y., Wang, L., Cao, F., and Tan, L. (2012). Identify schizophrenia using resting-state functional connectivity: an exploratory research and analysis. Biomed. Eng. Online 11, 1-1. doi:10.1186/1475-925X-11-50

Turner, M. R., Kiernan, M. C., Leigh, P. N., and Talbot, K. (2009). Biomarkers in amyotrophic lateral sclerosis. Lancet Neurol. 8, 94-109. doi:10. 1016/S1474-4422(08)70293-X

Turner, M. R., and Modo, M. (2010). Advances in the application of MRI to amyotrophic lateral sclerosis. Expert Opin. Med. Diagn. 4, 483-496. doi:10.1517/17530059.2010.536836

Vatolkin, I., Theimer, W., and Botteck, M. (2012). "Partition based feature processing for improved music classification," in Studies in Classification, Data Analysis, and Knowledge Organization, eds W. A. Gaul, A. Geyer-Schulz, L. Schmidt-Thieme, and J. Kunze (Berlin: Springer), 411419.

Verstraete, E., van den Heuvel, M. P., Veldink, J. H., Blanken, N., Mandl, R. C., Hulshoff Pol, H. E., et al. (2010). Motor network degeneration in amyotrophic lateral sclerosis: a structural and functional connectivity study. PLoS ONE 2012:e13664. doi:10.1371/ journal.pone.0013664

Verstraete, E., Veldink, J. H., Mandl, R. C. W., van den Berg, L. H., and van den Heuvel, M. P. (2011). Impaired structural motor connectome in amyotrophic lateral sclerosis. PLoS ONE 6:e24239. doi:10.1371/ journal.pone.0024239

Wang, S., and Melhem, E. R. (2005) Amyotrophic lateral sclerosis and primary lateral sclerosis: the role of diffusion tensor imaging and other advanced MR-based techniques as objective upper motor neuron markers. Ann. N. Y. Acad. Sci. 1064, 61-77. doi:10.1196/ annals. 1340.013

Wang, S., and Summers, R. M. (2012). Machine learning and radiology. Med. Image Anal. 16, 933-951. doi:10.1016/j.media.2012.02.005

Welsh, R. C., Chen, A. C., and Taylor, S. F. (2010). Low-frequency BOLD fluctuations demonstrate altered thalamocortical connectivity in schizophrenia. Schizophr. Bull. 36, 713-722. doi:10.1093/schbul/sbn145

Yu, Y., Shen, H., Zhang, H., Zeng, L.-L., Xue, Z., and Hu, D. (2013). Functional connectivity-based signatures of schizophrenia revealed by multiclass pattern analysis of resting-state fMRI from schizophrenic patients and their healthy siblings. Biomed. Eng. Online 12, 1-1. doi:10.1186/ 1475-925X-12-10 
Zeng, L. L., Shen, H., Liu, L., Wang, L., Li, B., Fang, P., et al. (2012). Identifying major depression using wholebrain functional connectivity: a multivariate pattern analysis. Brain 135, 1498-1507. doi:10.1093/brain/ aws 059

Zoccolella, S., Beghi, E., Palagano, G., Fraddosio, A., Samarelli, V., Lamberti, P., et al. (2006). Predictors of delayin the diagnosis and clinical trial entry of amyotrophic lateral sclerosis patients: a population-based study. J. Neurol. Sci. 250, 45-49. doi:10.1016/j.jns.2006.06.027

Conflict of Interest Statement: The authors declare that the research was conducted in the absence of any commercial or financial relationships that could be construed as a potential conflict of interest.

Received: 25 January 2013; accepted: 20 May 2013; published online: 10 June 2013.

Citation: Welsh RC, Jelsone-Swain LM and Foerster BR (2013) The utility of independent component analysis and machine learning in the identification of the amyotrophic lateral sclerosis diseased brain. Front. Hum. Neurosci. 7:251. doi: 10.3389/fnhum.2013.00251

Copyright (c) 2013 Welsh, Jelsone-Swain and Foerster. This is an open-access article distributed under the terms of the Creative Commons Attribution License, which permits use, distribution and reproduction in other forums, provided the original authors and source are credited and subject to any copyright notices concerning any third-party graphics etc. 